A. Usov ${ }^{1}$, DSc, Prof.,

Y. Morozov ${ }^{1}$, PhD, Assoc. Prof.,

M. Kunitsyn ${ }^{1}$,

A. Tonkonozhenko ${ }^{2}$,

I. Chernush ${ }^{2}$

${ }^{1}$ Odessa National Polytechnic University, 1 Shevchenko Ave., Odessa, Ukraine, 65044; e-mail: usov.anatolij.ua@gmail.com, moro-

zovyu@gmail.com, m.v.kunitsyn@opu.ua

${ }^{2}$ KB Yuzhnouye, 3 Krivorozhskay Ave., Dnepr, Ukraine,49008; e-mail: info@yuzhnouye.com

\title{
INVESTIGATION OF THE INFLUENCE OF STRUCTURAL INHOMOGENEITIES ON THE STRENGTH OF WELDED JOINTS OF FUNCTIONALLY GRADIENT MATERIALS
}

\begin{abstract}
А.В. Усов, Ю.О. Морозов, М.В. Куніцин, А.М. Тонконоженко, І.О. Чернии. Дослідження впливу структурних неоднорідностей на міцність зварних з’єднань функціонально-градієнтних матеріалів. Розглядається вплив дефектів на механічні властивості з'єднань функціонально-градієнтних матеріалів, який залежить від їх форми, умов експлуатації, та характеру навантаження. Наявність дефектів у зварних з'єднаннях суттєво знижують робочу здатність конструкцій, а при певних умовах можуть привести до їх руйнування. Проблема міцності конструкцій, які мають зварні з'єднання пов'язана з встановленням локальних концентраторів напружень біля дефектів типу тріщин, в яких напруження значно перевищують середні значення. Для визначення міцності зварних з'єднань і встановлення вимог які пред’являються до їх якості побудована математична модель, яка дозволяс досліджувати вплив найбільш вірогідних дефектів на міцність з'єднань. Врахування дефектності, що мають місце у зварювальних з'єднаннях на несучу здатність конструкцій можна реалізувати основним критерієм локального руйнування $K_{l c}$ та дією експлуатаційних навантажень. По знайденому із моделі значенню коефіцієнта інтенсивності напружень та величині теплового потоку, що діє на конструкцію і завдяки якому у з'єднанні формуються напруження, можна визначити граничні значення розміру дефекту, при якому цей дефект залишиться у стані рівноваги без формування із нього магістральної тріщини. Адекватність одержаних теоретичним шляхом результатів досліджень перевірялась на міцності зварних з'єднань алюмінієвих сплавів з урахуванням наявності в них гарячих тріщин. Деформаційне зміцнення зварних з'єднань, яке відбувається у високоміцних складних легованих алюмінієвих сплавів сприяє пониженню їх міцності в зв'язку з появою у них інтерметалідних фаз, а також не проварів.

Ключові слова: міцність зварного з’єднання, дефектність, математична модель, коефіцієнт інтенсивності напружень, адекватність

A. Usov, Yu. Morozov, M. Kunitsyn, A. Tonkonozhenko, I. Chernush. Investigation of the influence of structural inhomogeneities on the strength of welded joints of functionally gradient materials. The effect of defects on the mechanical properties of joints of functionally gradient materials, which depends on their shape, operating conditions, and the nature of the load, is considered. The presence of defects in the welded joints significantly reduces the working ability of the structures and, under certain conditions, can lead to their destruction. The problem of the strength of structures that have welded joints is because of the installation of local stress concentrators, near defects of the cracks in which the stresses are well above average. To determine the strength of welded joints and to establish the requirements for their quality, a mathematical model is built that allows you to investigate the impact of the most likely defects on the strength of the joints. The defects taking place in the welding joints on the load-bearing capacity of the structures can be realized by the necessary criterion of local destruction of $K_{I c}$ and the action of operational loads. Based on the value of the stress intensity factor and the value of the heat flux acting on the structure found by the model, by which the stresses are formed in the joint, it is possible to determine the limiting values of the defect size, at which this defect will remain in equilibrium without forming the main crack. The adequacy of the research results obtained was theoretically verified by the strength of welded joints of aluminum alloys, taking into account hot cracks in them. The deformation strengthening of welded joints, which occurs in high-strength complex alloyed aluminum alloys, reduces their strength because of the appearance of intermetallic phases in them, and welds.

Keywords: weld strength, defect, mathematical model, stress intensity factor, adequacy
\end{abstract}

\section{Introduction}

Various technological operations contribute to the occurrence of hereditary defects in the surface layer of products. These defects, being stress concentrators, contribute to crack formation, and, to the loss of the bearing capacity of structural elements both during their manufacturing and during operation.

Especially significant losses in the national economy from marriage because of hereditary defects that form in products during their manufacture and the operation of these products.

DOI: 10.15276/opu.1.60.2020.03

(c) 2020 The Authors. This is an open access article under the CC BY license (http://creativecommons.org/licenses/by/4.0/). 
Reducing defects in the manufacture of structural elements, improving the operational properties of such products, is an important national economic task, the solution of which leads to significant savings in material resources, labor and cost of them, and improving reliability during their operation.

\section{Analysis of recent research and publications}

The effect of defects on the mechanical properties of welded joints [1 -6] of functionally gradient materials is determined by the size and shape of the defects, their repetition rate, construction material, operating conditions and nature of the load [7, 8], therefore, the presence of defects in welded joints does not mean the loss of their performance. However, defects can significantly reduce the performance of structures and, under certain conditions, lead to their destruction. Therefore, to determine the reliability of welded structures and establish requirements for the quality of welded joints, it is necessary to have information about the influence of the most probable defects on the strength of joints. The greatest danger to structures is represented by internal defects since they must be detected without destroying the welded joint.

In structures operating under static and dynamic loads, the same defects unequally affect welded joints. Under a static load, the relative strength of the defect has the main effect on the strength of structures operating at temperatures down to $-60^{\circ} \mathrm{C}$, provided that the material of the welded joint has a large plasticity margin. At lower temperatures, the strength is characterized by the intensity of the stresses in the defect zone. Under dynamic loads, the strength of welded joints is determined by their resistance to fatigue stresses $[9,10]$. Undercuts, pores, slag inclusions, and lack of penetration (and/or lack of fusion) reduce the durability of structures, being the causes of the formation of stress concentration. Cracks of any size are not allowed in welded joints, as they contribute to the concentration of internal stresses, quickly spreading deep into the metal.

Lack of penetration (and/or lack of fusion) has a significant effect on the impact strength of the weld metal. According to [7], a lack of penetration (and/or lack of fusion) of $10 \%$ of the thickness of a welded joint can reduce fatigue strength by half, and a lack of penetration (and/or lack of fusion) of $40 . .50 \%$ reduces the endurance limits of steel by 2.5 times.

The operation of welded structures shows that welding stresses and strains contribute to a decrease in the bearing capacity of structures [9]. Sometimes, changing the size and shape of the welded structure reduces its performance, spoils the appearance, and can even lead to destruction. There is a general pattern of reduction in the strength of welded structures under the influence of shock in the presence of undercuts, pores, slag inclusions, and lack of penetration. The types, quantity, and sizes of permissible internal defects depend on the design purpose.

\section{The purpose of research}

To build a mathematical model that allows us to study the effect of defects in welded joints on the strength of welded structures and to determine the requirements for their quality, and to verify its adequacy by the experimental method.

\section{Statement of the main material}

Consider an infinite composite cylindrical body (Fig. 1) comprising three thin-walled transversally isotropic hollow cylinders, one of which is finite, $2 h$ high, models a weld. Between the cylinders, the full adhesion conditions are satisfied, and inside the weld, there are flat defects of an arbitrary nature (such as cracks, delaminated, and not delaminated inclusions) [11 - 17].

To determine the stress intensity factor under tensile load, it is necessary to construct a discontinuous solution [14] for a piecewise-homogeneous transversely isotropic space. Consider a space consisting of two different transversally isotropic half-spaces fully linked along the $z=0$ plane. On an arbitrary piecewise continuous surface $\Omega$, defects of an arbitrary nature are located (such as cracks, exfoliated and non-exfoliated inclusions).

Stress and displacement components:

$$
\begin{gathered}
\boldsymbol{\sigma}=\sigma_{k}(x, y, z)_{k=1}^{6}=\sigma_{x}, \sigma_{y}, \sigma_{z}, \tau_{y z}, \tau_{x z}, \tau_{x y}, \\
\mathbf{u}=u_{k}(x, y, z)_{k=1}^{3}=u, v, w .
\end{gathered}
$$


When $(x, y, z) \notin \Omega$ satisfy the generalized Hooke law:

$$
\begin{gathered}
\partial_{k} u_{k}=\sum_{j=1}^{3} s_{j k} \sigma_{j}, \quad k=1,2,3, \partial_{3} u_{2}+\partial_{2} u_{3}=s_{44} \sigma_{4}, \\
\partial_{1} u_{3}+\partial_{3} u_{1}=s_{44} \sigma_{5}, \partial_{2} u_{1}+\partial_{1} u_{2}=s_{66} \sigma_{6} .
\end{gathered}
$$

And equilibrium conditions:

$$
\begin{aligned}
& \partial_{1} \sigma_{1}+\partial_{2} \sigma_{6}+\partial_{3} \sigma_{5}+F_{1}=0, \\
& \partial_{1} \sigma_{6}+\partial_{2} \sigma_{2}+\partial_{3} \sigma_{4}+F_{2}=0, \\
& \partial_{1} \sigma_{5}+\partial_{2} \sigma_{4}+\partial_{3} \sigma_{3}+F_{3}=0 .
\end{aligned}
$$

Where $s_{k j}\left(x_{3}\right)=s_{j k}\left(x_{3}\right)=\left\{\begin{array}{l}s_{k j}^{+}, \text {at } x_{3}>0 ; \\ s_{k j}^{-}, \text {at } x_{3}<0,\end{array} \quad \partial_{1}=\frac{\partial}{\partial x}, \quad \partial_{2}=\frac{\partial}{\partial y}, \quad \partial_{3}=\frac{\partial}{\partial z}, \quad F_{k}, k=\overline{1,3}\right.$ are the components of the volume forces, $s_{k j}^{ \pm}$- the coefficients of the generalized Hooke law of a transversely isotropic medium, respectively, for the upper $\left(x_{3}>0\right)$ and lower $\left(x_{3}<0\right)$ half-space.

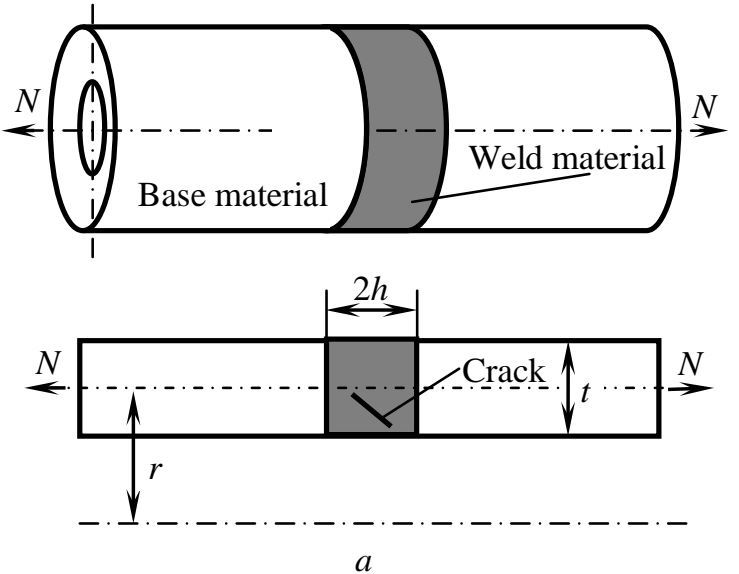

Fig. 1. Design scheme for determining the influence of defects located in a weld on the bearing capacity of cylindrical composite structures: welded body of a cylindrical structure (a); diagram of the power load on the element of the cylindrical structure (b)

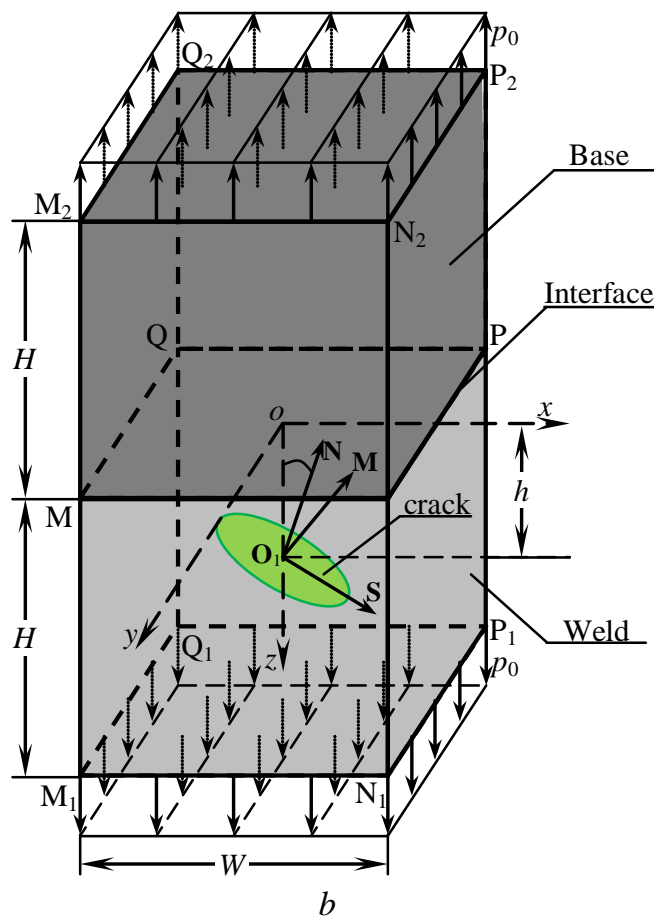

$b$

To write the conditions on the surface $\Omega$, where discontinuities of all components of the vectors $\sigma$ and $\mathbf{u}$ are possible, we introduce at each point on the surface $\Omega$ the local coordinate systems $(N, M, S)$ Fig. $1 \mathrm{~b}$. To do this, draw a tangent plane $P$ and a normal vector $\mathbf{n}$ to it at each point on the surface. The direction of the $N$ axis coincides with the direction of the $\mathbf{n}$ vector. We choose the other two axes $M, S$ mutually perpendicular to the plane $P$ so that after the rotation of the axes, the directions of the axes $(N, M, S)$ coincide with the direction of the corresponding axes $(Z, X, Y)$. In the new coordinate system of stress and displacement, we denote this:

$$
\begin{gathered}
\boldsymbol{\sigma}_{N}=\left\{\tilde{\sigma}_{k}(x, y, z)\right\}_{k=1}^{6}=\left\{\sigma_{N}, \sigma_{S}, \sigma_{Z}, \tau_{S Z}, \tau_{Z N}, \tau_{N S}\right\}, \\
\mathbf{u}_{N}=\left\{\tilde{u}_{k}\right\}_{k=1}^{3}=\left\{u_{M}, v_{S}, w_{N}\right\} .
\end{gathered}
$$
known:

Depending on the contact interaction with the surface space, six of the following values may be 


$$
\begin{gathered}
\tilde{\chi}^{ \pm}=\left\{\tilde{\chi}_{k}^{ \pm}\right\}_{k=1}^{6}, \tilde{\chi}_{k}^{ \pm}=\tilde{\zeta}_{k}^{+}\left(x_{1}, x_{2}, x_{3}\right) \pm \tilde{\zeta}_{k}^{-}\left(x_{1}, x_{2}, x_{3}\right),\left(x_{1}, x_{2}, x_{3}\right) \in \Omega, \\
\tilde{\zeta}_{k=1}^{ \pm 6}=\left\{\sigma_{3}^{ \pm}\left(x_{1}, x_{2}, x_{3}\right), \sigma_{5}^{ \pm}\left(x_{1}, x_{2}, x_{3}\right), \sigma_{6}^{ \pm}\left(x_{1}, x_{2}, x_{3}\right), u_{1}^{ \pm}\left(x_{1}, x_{2}, x_{3}\right), u_{2}^{ \pm}\left(x_{1}, x_{2}, x_{3}\right), u_{3}^{ \pm}\left(x_{1}, x_{2}, x_{3}\right)\right\}, \\
\left(\sigma_{k}^{ \pm}, u_{k}^{ \pm}\right)=\lim _{x_{i}^{ \pm} \rightarrow x_{i}}\left(\sigma_{k}^{ \pm}\left(x_{1}^{ \pm}, x_{2}^{ \pm}, x_{3}^{ \pm}\right), u_{k}^{ \pm}\left(x_{1}^{ \pm}, x_{2}^{ \pm}, x_{3}^{ \pm}\right)\right), i=\overline{1,3} .
\end{gathered}
$$

Point $\left(x^{+}, y^{+}, z^{+}\right)$on the normal side $\mathbf{n}$, and point $\left(x^{-}, y^{-}, z^{-}\right)$is on the opposite side. For definiteness, on the surface $\Omega$ we assume the following jumps to be known:

$$
\tilde{\chi}_{k}^{-}=\tilde{\zeta}_{k}^{+}(x, y, z)-\tilde{\zeta}_{k}^{-}(x, y, z), k=\overline{1,6},(x, y, z) \in \Omega
$$

The solution of the boundary value problem (2), (3), (5), (6) must be sought in the class $C_{0, s}^{1}\left(\mathbb{R}^{3}\right) \cap L_{1}\left(\mathbb{R}^{3}\right)$, where $C_{0, s}^{1}$ is the space of functions continuous in all derivatives up to the $m$-th order with the exception of the surface $\Omega, L_{1}\left(\mathbb{R}^{3}\right)$ is the space of functions integrable in $\mathbb{R}^{3}$.

We introduce the notation:

$$
\begin{gathered}
\mathbf{v}=\left\{\mathrm{v}_{k}(x, y, z)\right\}_{k=1}^{9}, \mathrm{v}_{k}=\sigma_{k}, k=\overline{1,6}, v_{k+6}=u_{k}, k=\overline{1,3}, \\
\tilde{\mathbf{v}}=\left\{\tilde{v}_{k}(x, y, z)\right\}_{k=1}^{9}, \tilde{v}_{k}=\tilde{\sigma}_{k}, k=\overline{1,6}, \tilde{v}_{k+6}=\tilde{u}_{k}, k=\overline{1,3}, \\
\mathbf{D}_{9}\left[x_{3}, \partial_{1}, \partial_{2}, \partial_{3}\right]=\begin{array}{ccc}
\mathbf{D}_{0} & 0_{3 \times 3}, \\
-\mathbf{S}\left(x_{3}\right) & \mathbf{D}_{0}^{T},
\end{array} \\
\mathbf{f}=\left\{-F_{1},-F_{2},-F_{3}, \alpha_{1} T, \alpha_{2} T, \alpha_{3} T, 0,0,0\right\}, \\
\mathbf{D}_{0}=\left(\begin{array}{cccccc}
\partial_{1} & 0 & 0 & 0 & \partial_{3} & \partial_{2} \\
0 & \partial_{2} & 0 & \partial_{3} & 0 & \partial_{1} \\
0 & 0 & \partial_{3} & \partial_{2} & \partial_{1} & 0
\end{array}\right), \\
\mathbf{S}\left(x_{3}\right)=\left\{s_{k j}\left(x_{3}\right)\right\}_{k, j=\overline{1,6}}, \\
\mathbf{S}\left(x_{3}\right)=\left(\begin{array}{cccccc}
s_{11} & s_{12} & s_{13} & 0 & 0 & 0 \\
s_{12} & s_{11} & s_{13} & 0 & 0 & 0 \\
s_{13} & s_{13} & s_{33} & 0 & 0 & 0 \\
0 & 0 & 0 & s_{44} & 0 & 0 \\
0 & 0 & 0 & 0 & s_{44} & 0 \\
0 & 0 & 0 & 0 & 0 & s_{66}
\end{array}\right),
\end{gathered}
$$

Then equations (2), (3) can be represented as:

$$
\mathbf{D}\left[x_{3}, \partial_{1}, \partial_{2}, \partial_{3}\right] \mathbf{v}=\mathbf{f},\left(x_{1}, x_{2}, x_{3}\right) \notin \Omega .
$$

We continue the matrix equation (7) to the entire space. To do this, using the properties of the generalized functions of slow growth $\mathfrak{I}^{\prime}\left(\mathbb{R}^{3}\right)$, we continue the desired functions to the space $\mathfrak{I}^{\prime}\left(\mathbb{R}^{3}\right)$ whose singularity carrier comprises the surface $\Omega$, i.e., suppsing $=\Omega$.

Given the relationship between conventional and generalized $\tilde{\partial}_{k}(k=1,2,3)$ derivatives:

$$
\begin{gathered}
\partial_{j} v_{k}=\tilde{\partial}_{j} v_{k}-\left\langle v_{k}\right\rangle_{\Omega}^{-} \kappa_{j} \delta(\Omega)+\delta_{3 j} \chi_{k}^{-}(x, y), j=\overline{1,3}, k=\overline{1,9}, \\
\kappa_{1}=\cos (N, X), \kappa_{2}=\cos (N, Y), \kappa_{3}=\cos (N, Z),
\end{gathered}
$$

where $\tilde{\partial}_{j}, j=\overline{1,3}$ are the differentiation operators in the space $\mathfrak{I}^{\prime}\left(\mathbb{R}^{3}\right), \delta(\Omega)$ is the generalized Dirac function concentrated on the surface $\Omega$. Taking into account formulas (8), we reduce the boundaryvalue problem (5), (6), (7) to the following boundary-value problem in the space $\mathfrak{I}^{\prime}\left(\mathbb{R}^{3}\right)$ : 


$$
\begin{gathered}
\mathbf{D}\left[z, \tilde{\partial}_{1}, \tilde{\partial}_{2}, \tilde{\partial}_{3}\right] \mathbf{v}=\tilde{\mathbf{f}}, \\
v_{k}^{+}=v_{k}^{-}, k=3,4,5,7,8,9,
\end{gathered}
$$

where $v_{k}^{ \pm} \in \mathfrak{I}_{ \pm}^{\prime}\left(\mathbb{R}^{3}\right), \tilde{\mathbf{f}}=\left\{\tilde{f}_{j}\right\}_{j=1}^{9}$;

$$
\begin{aligned}
& \tilde{f}_{1}=\left(\left\langle v_{1}\right\rangle_{\Omega}^{-} \kappa_{1}+\left\langle v_{5}\right\rangle_{\Omega}^{-} \kappa_{3}+\left\langle v_{6}\right\rangle_{\Omega}^{-} \kappa_{2}\right) \delta(\Omega)-F_{1} ; \\
& \tilde{f}_{2}=\left(\left\langle v_{2}\right\rangle_{\Omega}^{-} \kappa_{2}+\left\langle v_{4}\right\rangle_{\Omega}^{-} \kappa_{3}+\left\langle v_{6}\right\rangle_{\Omega}^{-} \kappa_{1}\right) \delta(\Omega)-F_{2} ; \\
& \tilde{f}_{3}=\left(\left\langle v_{3}\right\rangle_{\Omega}^{-} \kappa_{3}+\left\langle v_{4}\right\rangle_{\Omega}^{-} \kappa_{2}+\left\langle v_{5}\right\rangle_{\Omega}^{-} \kappa_{1}\right) \delta(\Omega)-F_{3} ; \\
& \tilde{f}_{4}=\left\langle v_{7}\right\rangle_{\Omega}^{-} \kappa_{1} \delta(\Omega) ; \\
& \tilde{f}_{5}=\left\langle v_{8}\right\rangle_{\Omega}^{-} \kappa_{2} \delta(\Omega) ; \\
& \tilde{f}_{6}=\left\langle v_{9}\right\rangle_{\Omega}^{-} \kappa_{3} \delta(\Omega) ; \\
& \tilde{f}_{7}=\left(\left\langle v_{8}\right\rangle_{\Omega}^{-} \kappa_{3}+\left\langle v_{9}\right\rangle_{\Omega}^{-} \kappa_{2}\right) ; \\
& \tilde{f}_{8}=\left(\left\langle v_{7}\right\rangle_{\Omega}^{-} \kappa_{3}+\left\langle v_{9}\right\rangle_{\Omega}^{-} \kappa_{1}\right) \delta(\Omega) ; \\
& \tilde{f}_{9}=\left(\left\langle v_{7}\right\rangle_{\Omega}^{-} \kappa_{2}+\left\langle v_{8}\right\rangle_{\Omega}^{-} \kappa_{1}\right) \delta(\Omega) .
\end{aligned}
$$

The $w_{j}=\left\{w_{k j}\left(x, y, z, x_{0}, y_{0}, z_{0}\right)\right\}_{k=1, \overline{1}}, w_{k j} \in \mathfrak{I}^{\prime}\left(\mathbb{R}^{3}\right), j=\overline{1,9}$ vector system is called the fundamental solutions of a discontinuous (FSD) for a piecewise homogeneous transversely isotropic space if they satisfy the following system of boundary value problems:

$$
\left\{\begin{array}{c}
D\left[x_{3}, \tilde{\partial}_{1}, \tilde{\partial}_{2}, \tilde{\partial}_{3}\right] w=\tilde{\mathbf{f}}_{0 j} ; \\
w_{k}^{+}=w_{k}^{-}, k=3, \overline{5,9}, w_{k}^{ \pm} \in \mathfrak{I}^{\prime}\left(\mathbb{R}^{3}\right), \quad j=\overline{1,9},\left(x_{1}, x_{2}, x_{3}\right) \in \mathbb{R}^{3}, \\
\tilde{\mathbf{f}}_{0 j}=\left\{f_{k j}^{0}\right\}_{k=1,9}=\left\{\delta_{k j}\right\}_{k=1,9} \delta\left(x_{1}-x_{1}^{0}, x_{2}-x_{2}^{0}, x_{3}-x_{3}^{0}\right) .
\end{array}\right.
$$

If the fundamental solution is found, then the discontinuous solution is obtained using convolution:

$$
\mathrm{v}_{k}=\sum_{j=1}^{9} w_{k j} \cdot \tilde{f}_{j}=\sum_{j=1}^{9} \iint_{\mathbb{R}^{3}} w_{k j}\left(\mathbf{x}, \mathbf{x}^{0}\right) \tilde{f}_{j}\left(x^{0}\right) d \mathbf{x}^{0}, \quad \mathbf{x}=\left(x_{1}, x_{2}, x_{3}\right), \mathbf{x}^{0}=\left(x_{1}^{0}, x_{2}^{0}, x_{3}^{0}\right) .
$$

Applying the three-dimensional Fourier transform to (11), we obtain with respect to $\mathbf{W}_{k j}^{ \pm}\left(\alpha_{1}, \alpha_{2}, \alpha_{3}\right)=\mathbf{F}_{3}\left[w_{k}^{ \pm}\right]$with respect to the variable $\alpha_{3}$ the Riemann matrix boundary value problem in the space $\mathfrak{I}^{\prime}\left(\mathbb{R}^{3}\right)$ :

$$
\begin{gathered}
\mathbf{M}_{+} \mathbf{W}_{j}^{+}=\mathbf{M}_{-} \mathbf{W}_{j}^{-}+\mathbf{f}_{*_{j}}, \\
\mathbf{M}_{ \pm}= \pm \mathbf{D}\left[ \pm 0,-i \alpha_{1},-i \alpha_{2},-i \alpha_{3}\right], \\
\mathbf{f}_{*_{j}}=\left\{f_{*_{j}}\right\}=\left\{\delta_{k j} e_{0}^{*}\right\} ; e_{0}^{*}=e^{i \alpha x_{1}^{0}+i \alpha x_{2}^{0}+i \alpha x_{3}^{0}} ; \mathbf{W}_{j}^{ \pm}=\left\{W_{k j}^{ \pm}\right\}_{k=1}^{9} .
\end{gathered}
$$

We will carry out all further calculations for the case when the surface $\Omega$ is a plane defect parallel to the plane $x_{3}=0$, i.e., when $\kappa_{1}=\kappa_{2}=0$ and $\kappa_{3}=1$, therefore $\tilde{f}_{j}=0, j=4,5,9$ and $\mathbf{f}_{*_{j}}=0$, $j=4,5,9$, respectively.

Using the theorems from [1] we obtain the representation:

$$
\begin{gathered}
e_{0}^{*}=e_{0}^{+}+e_{0}^{-}, \\
\left(\left(-i \alpha_{3}\right) \mathrm{W}_{k j}=\left(\left(-i \alpha_{3}\right) \mathrm{W}_{k j}^{+}+\frac{1}{2} \chi_{k 0}^{+}\right)-\left(\left(-i \alpha_{3}\right) \mathrm{W}_{k j}^{-}-\frac{1}{2} \chi_{k 0}^{+}\right)\right),
\end{gathered}
$$

where $\chi_{k 0}^{+}, k=\overline{1,9}, k \neq 4,5,9$ - sums of transformants of functions $w_{k j}$ at $x_{3}=0$.

Then, taking into account (14) and using Theorem 2.2 from [1], we obtain: 


$$
\begin{gathered}
\mathbf{M}_{ \pm}\left(-i \alpha_{2},-i \alpha_{3}\right) \mathbf{W}_{j}^{ \pm}=\mathbf{T}_{j}^{ \pm}, \\
\mathbf{T}_{j}^{ \pm}=\left\{t_{k j}^{ \pm}\right\}_{k=1,9}=\theta\left( \pm z_{0}\right) e_{0}^{ \pm}\left\{\delta_{k j}\right\} \mp \frac{1}{2} \boldsymbol{f}_{0}^{+},
\end{gathered}
$$

where $\left\{\zeta_{k}\right\}_{k=1,9}=f_{0}^{+}=\left\{\chi_{50}^{+}, \chi_{40}^{+}, \chi_{30}^{+}, 0.0, \chi_{90}^{+}, \chi_{80}^{+}, \chi_{70}^{+}, 0\right\}$.

To solve equations (15), we introduce the following combinations:

$$
\begin{gathered}
W_{7 j}^{ \pm}=-\left(-i \alpha_{2}\right) \psi_{1 j}^{ \pm}-\left(-i \alpha_{1}\right) \psi_{2}^{ \pm}, W_{8 j}^{ \pm}=\left(-i \alpha_{1}\right) \psi_{1 j}^{ \pm}-\left(-i \alpha_{2}\right) \psi_{2 j}^{ \pm}, \\
W_{5 j}^{ \pm}=-\left(-i \alpha_{2}\right) \tau_{1 j}^{ \pm}-\left(-i \alpha_{1}\right) \tau_{2 j}^{ \pm}, W_{4 j}^{ \pm}=\left(-i \alpha_{1}\right) \tau_{1 j}^{ \pm}-\left(-i \alpha_{2}\right) \tau_{2 j}^{ \pm},
\end{gathered}
$$

where $\psi_{k j}^{ \pm}, \tau_{k j}^{ \pm}, k=1,2$ are new unknown functions.

Then the system of equations (15) decomposes into two independent systems:

$$
\begin{aligned}
& \mathbf{L}_{ \pm}\left(-i \alpha_{3}, r^{2}\right) \mathbf{W}_{j}^{(1) \pm}=\mathbf{T}_{j}^{(1) \pm}, \\
& \mathbf{G}_{ \pm}\left(-i \alpha_{3}, r^{2}\right) \mathbf{W}_{j}^{(2) \pm}=\mathbf{T}_{j}^{2 \pm},
\end{aligned}
$$

where $\mathbf{L}_{ \pm}\left(-i \alpha_{3}, r^{2}\right)=\left(\begin{array}{cc}-c_{66}^{ \pm} r^{2} & \left(-i \alpha_{3}\right) \\ -\left(-i \alpha_{3}\right) & 1 / c_{44}^{ \pm}\end{array}\right)$;

$$
\begin{aligned}
& \mathbf{W}_{j}^{(1) \pm}=\left\{w_{l j}^{(1) \pm}\right\}_{l=1,2}=\left\{r^{2} \psi_{1 j}^{ \pm}, r^{2} \tau_{1 j}^{ \pm}\right\} ; \\
& \mathbf{T}_{j}^{(1) \pm}=\left\{\left(-i \alpha_{2}\right) t_{1 j}^{ \pm}-\left(-i \alpha_{1}\right) t_{2 j}^{ \pm},\left(-i \alpha_{1}\right) t_{7 j}^{ \pm}-\left(-i \alpha_{2}\right) t_{8 j}^{ \pm}\right\} ; \\
& \mathbf{W}_{j}^{(2) \pm}=\left\{w_{l j}^{(2) \pm}\right\}_{l=1,4}=\left\{r^{2} \tau_{2 j}^{ \pm}, w_{3 j}^{ \pm}, w_{9 j}^{ \pm}, r^{2} \psi_{2 j}^{ \pm}\right\} ; \\
& \mathbf{T}_{j}^{(2) \pm}=\left\{\left(-i \alpha_{1}\right) t_{1 j}^{ \pm}+\left(-i \alpha_{2}\right) t_{2 j}^{ \pm}, t_{3 j}^{ \pm}, t_{6 j}^{ \pm},\left(-i \alpha_{2}\right) t_{7 j}^{ \pm}+\left(-i \alpha_{1}\right) t_{8 j}^{ \pm}\right\} ; \\
& \mathbf{G}_{ \pm}\left(-i \alpha_{3}, r^{2}\right)=\left(\begin{array}{cccc}
\left(-i \alpha_{3}\right) & -\frac{c_{13}^{ \pm}}{c_{33}^{ \pm}} r^{2} & 0 & -\left(c_{11}^{ \pm}-\frac{c_{13}^{ \pm}}{c_{33}^{ \pm}}\right) \\
1 & \left(-i \alpha_{3}\right) & 0 & 0 \\
0 & -\frac{1}{c_{33}^{ \pm}} & \left(-i \alpha_{3}\right) & \frac{c_{13}^{ \pm}}{c_{33}^{ \pm}} \\
-\frac{1}{c_{44}^{ \pm}} & 0 & -r^{2} & \left(-i \alpha_{3}\right)
\end{array}\right), r^{2}=\alpha_{1}^{2}+\alpha_{2}^{2} .
\end{aligned}
$$

Directly from equations (17) we obtain:

$$
\begin{gathered}
\mathbf{W}_{j}^{(1) \pm}=\mathbf{L}_{ \pm}^{-1}\left(-i \alpha_{3}, r^{2}\right) \mathbf{T}_{j}^{(1) \pm}, \\
\mathbf{W}_{j}^{(2) \pm}=\mathbf{G}_{ \pm}^{-1}\left(-i \alpha_{3}, r^{2}\right) \mathbf{T}_{j}^{(2) \pm}, \\
\mathbf{L}_{ \pm}^{-1}=\left\{l_{i j}^{*, \pm}\right\}_{i, j=1,2}, \\
\mathbf{G}_{ \pm}^{-1}=\left\{g_{i j}^{*, \pm}\right\}_{i, j=1, \overline{1}} .
\end{gathered}
$$

In expanded form, equalities (18) are written:

$$
\begin{gathered}
\left\{r^{2} \psi_{1 j}^{ \pm}, r^{2} \tau_{1 j}^{ \pm}\right\}=\left\{\theta\left( \pm x_{3}^{0}\right) \frac{(-1)^{j+1}\left(-i \alpha_{1}\right)^{m_{j}}}{\left(-i \alpha_{2}\right)^{-n_{j}}} \frac{e_{0}^{ \pm}}{P_{1}^{ \pm}\left(\alpha_{3}, r\right)} \tilde{l}_{l p}^{*, \pm} \tilde{\delta}_{p j} \mp \frac{1}{2 P_{1}^{ \pm}\left(\alpha_{3}, r\right)} \sum_{k=1}^{2} l_{l k}^{*, \pm} \bar{\chi}_{k 0}^{+}\right\}_{l=1,2}, \\
\left\{r^{2} \tau_{2 j}^{ \pm}, w_{3 j}^{ \pm}, w_{9 j}^{ \pm} ; \quad r^{2} \Psi_{2 j}^{ \pm}\right\}=\left\{\theta\left( \pm x_{3}^{0}\right) \frac{\left(-i \alpha_{1}\right)^{n_{j}}}{\left(-i \alpha_{2}\right)^{-m_{j}}} \frac{e_{0}^{ \pm}}{P_{2}^{ \pm}\left(\alpha_{3}, r\right)} \tilde{g}_{l p}^{*, \pm} \tilde{\delta}_{p j} \mp \frac{1}{2 P_{2}^{ \pm}\left(\alpha_{3}, r\right)} \sum_{k=1}^{4} g_{l k}^{*, \pm} \tilde{\chi}_{k 0}^{+}\right\}_{l=\overline{1,4}},
\end{gathered}
$$

where $\left\{\tilde{l}_{l p}^{*, \pm}\right\}_{p=1,4}^{l=\overline{1,2}}=\left\{l_{l p}^{*, \pm}\right\}_{p=1,1,2,2}^{l=\overline{1,4}},\left\{\bar{\delta}_{p j}\right\}_{p=1, \overline{1,6}}=\left\{\delta_{p j}\right\}_{p=1,2,7,8}$; 


$$
\begin{aligned}
& l_{11}^{*, \pm}\left(\alpha_{3}, r\right)=-\frac{1}{c_{44}^{ \pm}}, l_{k 2}^{*, \pm}\left(\alpha_{3}, r\right)=\left(-i \alpha_{3}\right)^{2-k}\left(c_{66}^{ \pm} r^{2}\right)^{(k-1)}, k=1,2, l_{21}^{*, \pm}\left(\alpha_{3}, r\right)=-l_{12}^{*, \pm}\left(\alpha_{3}, r\right) \\
& P_{1}^{ \pm}\left(\alpha_{3}, r\right)=c_{66}^{ \pm} r^{2}+c_{44}^{ \pm} \alpha_{3}^{2}=c_{44}^{ \pm}\left(\alpha_{3}-Z^{ \pm} r\right)\left(\alpha_{3}-\bar{Z}^{ \pm} r\right), \quad Z^{ \pm}=i \frac{\sqrt{c_{44}^{ \pm} C_{66}^{ \pm}}}{c_{44}^{ \pm}} \\
& \left\{\bar{\chi}_{k 0}^{+}\right\}_{k=1,2}=\left\{\sum_{i=4}^{5}(-1)^{i}\left(-i \alpha_{i-3}\right) \chi_{i 0}^{+}, \sum_{i=7}^{8}(-1)^{i+1}\left(-i \alpha_{9-i}\right) \chi_{i 0}^{+}\right\} \text {; } \\
& n_{j}=\left\{\begin{array}{rr}
0 & j=2,7,3,6 \\
1 & j=1,8
\end{array}, m_{j}=\left\{\begin{array}{rr}
0 & j=1,8,3,6 \\
1 & j=2,7
\end{array},\left\{\tilde{g}_{l p}^{*, \pm}\right\}_{p=1,6}^{l=\overline{1,4}}=\left\{g_{l p}^{*, \pm}\right\}_{p=1,1,2,3,4,4}^{l=\overline{1,4}} ; ；\right.\right. \\
& \left\{\tilde{\delta}_{p j}\right\}_{p=\overline{1,6}}=\left\{\delta_{p j}\right\}_{p=1,2,3,6,7,8} \text {; } \\
& g_{11}^{ \pm}\left(\alpha_{3}, r^{2}\right)=-h_{1}^{ \pm} \lambda \frac{ \pm}{3}, g_{12}^{ \pm}\left(\alpha_{3}, r^{2}\right)=-h_{2}^{ \pm} \lambda_{1}^{ \pm}, g_{13}^{ \pm}\left(\alpha_{3}, r^{2}\right)=h_{3}^{ \pm} \lambda_{2}^{ \pm}, \quad g_{14}^{ \pm}\left(\alpha_{3}, r^{2}\right)=h_{4}^{ \pm} \lambda \lambda_{2}^{ \pm} ; \\
& h_{1}^{ \pm}=\left(-i \alpha_{3}\right), h_{k}^{ \pm}=\left(-i \alpha_{3}\right)^{k-2} r^{2}, k=2,3, h_{4}^{ \pm}=\left(-i \alpha_{3}\right)^{2}, \lambda_{l}^{ \pm}=\left(c_{13}^{ \pm} \alpha_{3}^{2}-c_{1 l}^{ \pm} r^{2}\right) c_{44}^{ \pm}, l=1,3 ; \\
& h_{1}^{ \pm}=\left(-i \alpha_{3}\right), h_{k}^{ \pm}=\left(-i \alpha_{3}\right)^{k-2} r^{2}, k=2,3, h_{4}^{ \pm}=\left(-i \alpha_{3}\right)^{2}, \lambda_{l}^{ \pm}=\left(c_{13}^{ \pm} \alpha_{3}^{2}-c_{11}^{ \pm} r^{2}\right) c_{44}^{ \pm}, \quad l=1,3 \text {; } \\
& \lambda_{2}^{ \pm}=\left(\bar{c}_{13}^{ \pm 2}-c_{13}^{ \pm 2}\right) r^{2}, \lambda_{4}^{ \pm}=\left(\bar{c}_{13}^{ \pm 2}-c_{13}^{ \pm 2}-c_{13}^{ \pm} c_{44}^{ \pm}\right) r^{2}+c_{33}^{ \pm} c_{44}^{ \pm} \alpha_{3}^{2}, \lambda_{5}^{ \pm}=\left(c_{13}^{ \pm}+c_{44}^{ \pm}\right) \text {; } \\
& \lambda_{6}^{ \pm}=\left(-i \alpha_{3}\right)^{-1}\left(c_{33}^{ \pm} \alpha_{3}^{2}+c_{44}^{ \pm} r^{2}\right), \lambda \frac{ \pm}{7}=\left(-i \alpha_{3}\right)^{-1}\left(c_{44}^{ \pm} \alpha_{3}^{2}+c_{11}^{ \pm} r^{2}\right) \text {; } \\
& P_{2}^{ \pm}\left(\alpha_{3}, r\right)=c_{33}^{ \pm} c_{44}^{ \pm} \alpha_{3}^{4}+\left(\bar{c}_{13}^{ \pm 2}+c_{44}^{ \pm 2}-\left(c_{13}^{ \pm}-c_{44}^{ \pm}\right)^{2}\right) r^{2} \alpha_{3}^{2}+c_{11}^{ \pm} c_{44}^{ \pm} r^{4}, \bar{c}_{13}^{ \pm 2}=c_{11}^{ \pm} c_{33}^{ \pm} ; \\
& \left\{\tilde{\chi}_{k 0}^{+}\right\}_{k=\overline{1,4}}=\left\{\sum_{i=4}^{5}\left(-i \alpha_{6-i}\right) \chi_{i 0}^{+}, \chi_{30}^{+}, \chi_{6,0}^{+}, \sum_{i=7}^{8}\left(-i \alpha_{i-6}\right) \chi_{i 0}^{+}\right\} \text {. }
\end{aligned}
$$

Inverting the Fourier transform regarding $\alpha_{3}$ and passing to the limit at $x_{3} \rightarrow \pm 0$, we obtain:

$$
\begin{gathered}
\left\{w_{l j}^{(1) \pm}\right\}_{l=1,2}=\theta\left( \pm x_{3}^{0}\right)(-1)^{j+1} \frac{\left(-i \alpha_{1}\right)^{n_{j}} r^{\theta_{j}}}{\left(-i \alpha_{2}\right)^{-m_{j}}}\left\{\tilde{f}_{1 l} \bar{S}_{l j}^{0, \pm} e^{\bar{Z}^{ \pm} r x_{3}^{0}}+\sum_{k=1}^{2} S_{l k}^{ \pm} \tilde{f}_{l k} \bar{\chi}_{k 0}^{+}\right\}_{l=1,2}, \\
\left\{w_{l j}^{(2) \pm}\right\}_{l=1,4}=\theta\left( \pm x_{3}^{0}\right) \frac{\left(-i \alpha_{1}\right)^{n_{j}} r^{\theta_{j}}}{\left(-i \alpha_{2}\right)^{-m_{j}}}\left\{f_{1 l} \sum_{n=1}^{3} R_{l j n}^{0, \pm} e^{i z_{n}^{ \pm} r x_{3}^{0}}-\sum_{k=1}^{4} \bar{R}_{l k}^{ \pm} f_{l k} \tilde{\chi}_{k 0}^{+}\right\}_{l=1,4},
\end{gathered}
$$

where $\theta_{j}=\left\{\begin{array}{cc}0 & j=1,2 \\ 1 & j=3,7,8 ; \\ 2 & j=6\end{array}\left\{f_{1 k}\right\}_{k=1,4}=\left\{1, r, r^{2}, r\right\}, R_{p k n}^{ \pm}=i \frac{\tilde{g}_{p k}^{*, \pm}\left(z^{ \pm}, 1\right)}{q_{n}^{ \pm}\left(z_{n}^{ \pm}\right) \bar{q}_{n}^{ \pm}\left(z_{n}^{ \pm}\right)}, \quad p, k=\overline{1,4} ; \quad R_{p k}^{ \pm}=\sum_{n=1}^{3} R_{p k n}^{ \pm}\right.$;

$$
\begin{aligned}
& \left\{R_{p j n}^{0,+}\right\}_{j=1,6}^{p=\overline{1,6}}=\left\{R_{p k n}^{+}\right\}_{p=1,6}^{k=\overline{1,6}} \operatorname{diag}\left\{\tilde{\delta}_{k j}\right\}_{j=1,6}^{k=\overline{1,6}}, q_{n}^{ \pm}\left(z_{n}^{ \pm}\right)=\prod_{l=1, l \neq n}^{2}\left(z_{n}^{ \pm}-z_{l}^{ \pm}\right), \bar{q}_{n}^{ \pm}\left(z_{n}^{ \pm}\right)=\prod_{l=1}^{2}\left(z_{n}^{ \pm}-\bar{z}_{l}^{ \pm}\right) ; \\
& \left\{S_{p j}^{0,+}\right\}_{j=1, \overline{1}}^{p=\overline{1,6}}=\left\{S_{p k}^{+}\right\}_{p=1,2}^{k=\overline{1,4}} \operatorname{diag}\left\{\bar{\delta}_{k j}\right\}_{j=\overline{1,4}}^{k=\overline{1,4}}, S_{p k}^{ \pm}=i \frac{\tilde{l}_{p k}^{* \pm}\left(z^{ \pm}, 1\right)}{q^{ \pm}\left(z_{n}^{ \pm}\right)}, \quad p=1,2 ; k=\overline{1,4} ; \\
& \left\{\tilde{f}_{1 k}\right\}_{k=1,2}=\left\{r^{-1} \tilde{f}_{2 k}\right\}_{k=1,2},\left\{\tilde{f}_{2 k}\right\}_{k=1,2}=\{1, r\}, q^{ \pm}\left(z^{ \pm}\right)=z^{ \pm}-\bar{z}^{ \pm}, \bar{q}^{ \pm}\left(z^{ \pm}\right)=\bar{z}^{ \pm}-z^{ \pm} .
\end{aligned}
$$

Equations (19), (20) contain six unknown functions. To determine them, we use conditions (10) with respect to the variable $x_{3}$. As a result, we get the idea:

$$
\begin{gathered}
\left\{\bar{\chi}_{10}^{+}, r \bar{\chi}_{20}^{+}\right\}=\tilde{\mathbf{A}}_{0}^{-1} \mathbf{b}_{j}, \tilde{\mathbf{A}}_{0}^{-1}=\left\{\tilde{a}_{k j}^{*}\right\}, \tilde{\mathbf{A}}_{0}=\overline{\mathbf{H}}^{+}-\mathbf{H}^{-}, \mathbf{H}^{ \pm}= \pm\left\{S_{p k}^{ \pm}\right\}_{p=1,2}^{k=1,2}, \\
\mathbf{b}_{j}=\left\{(-1)^{j+1} \frac{\left(-i \alpha_{1}\right)^{m_{j}} r^{\theta_{j}}}{\left(-i \alpha_{2}\right)^{-n_{j}}} e^{i \alpha_{1} x_{1}^{0}+i \alpha_{2} x_{2}^{0}}\left(\theta\left(+x_{3}^{0}\right) S_{p j}^{0,+} e^{i z^{+} r x_{3}^{0}}-\theta\left(-x_{3}^{0}\right) \bar{S}_{p j}^{0,-} e^{\overline{\bar{z}}-r x_{3}^{0}}\right)\right\}_{p=1,2},
\end{gathered}
$$




$$
\begin{gathered}
\left\{\tilde{\chi}_{10}^{+}, r \tilde{\chi}_{20}^{+}, r^{2} \tilde{\chi}_{30}^{+}, r \tilde{\chi}_{40}^{+}\right\}=\mathbf{A}_{0}^{-1} \mathbf{d}_{j} ; \mathbf{A}_{0}^{-1}\left\{a_{k j}^{*}\right\}_{k, j=\overline{1,4}}=\overline{\mathbf{N}}^{+}-\mathbf{N}^{-}, \mathbf{N}^{ \pm}= \pm\left\{R_{p k}^{ \pm}\right\}_{k=\overline{1,4}, \overline{1,4}}, \\
\mathbf{d}_{j}=\left\{\frac{\left(-i \alpha_{1}\right)^{n_{j}} r^{\theta_{j}}}{\left(-i \alpha_{2}\right)^{-m_{j}}} e^{-i \alpha_{1} x_{1}^{0}-i \alpha_{2} x_{2}^{0}} \sum_{n=1}^{3}\left(\theta\left(x_{3}^{0}\right) R_{p j n}^{0,+} e^{i z_{n}^{+} x_{3}^{0}}+\theta\left(-x_{3}^{0}\right) \bar{R}_{p j n}^{0,-} e^{i \overline{\bar{z} m} \times x_{3}^{0}}\right)\right\}_{p=1,4}
\end{gathered}
$$

Applying the inverse two-dimensional Fourier transform to (18), taking into account (21), (22), we obtain the representation for the fundamental discontinuous solution:

$$
w_{k j}\left(\boldsymbol{x}, \boldsymbol{x}^{0}\right)=\theta\left(+x_{3}^{0}\right) w_{k j}^{+}+\theta\left(-x_{3}^{0}\right) w_{k j}^{-}, k=3,9 .
$$

The found fundamental discontinuous solution (23) allows using convolution (12) to obtain the desired discontinuous solution.

Let a disc-shaped elliptic crack $z=h$ be contained in a composite transversely isotropic space in the $\Omega:\left\{\frac{x_{1}^{2}}{a^{2}}+\frac{x_{1}^{2}}{b^{2}}<1\right\}$ plane, to the banks of which a constant load is applied i.e. $f_{3}^{ \pm}(x, y)=-P$, $f_{k}^{ \pm}(x, y)=0, k=4,5$.

$$
\begin{gathered}
\sum_{k=7}^{8} R_{2 k}^{+} \partial_{k-6} \chi_{k}^{-}+\frac{1}{\pi} \iint_{\Omega} R_{26}^{+} \chi_{9}^{-} \frac{1}{r_{0}^{3}} d \boldsymbol{x}_{0}-\frac{1}{\pi} \sum_{k=5}^{7} \iint_{\Omega} \chi_{14-k}^{-} \sum_{n=1}^{3} \beta_{2 k n}^{++} \tilde{K}_{n, k+1}^{1,+}\left(\boldsymbol{x}-\boldsymbol{x}^{0}, \bar{z}_{2, n}^{+}\right) d \boldsymbol{x}_{0}=P_{3}, \\
-\left[\bar{S}_{24}^{+}+\bar{R}_{16}^{+}\right] \frac{1}{\pi} \iint_{\Omega} \tilde{\chi}_{7}^{-} \partial_{12}^{2} \frac{1}{r_{0}} d \boldsymbol{x}^{0}+\frac{1}{\pi} \iint_{\Omega} \tilde{\chi}_{8}^{-}\left[\bar{S}_{23}^{+} \partial_{1}^{2} \frac{1}{r_{0}}-\bar{R}_{15}^{+} \partial_{2}^{2} \frac{1}{r_{0}}\right] d \boldsymbol{x}^{0}-\bar{R}_{14}^{+} \partial_{2} \tilde{\chi}_{9}^{-}+ \\
+\frac{1}{\pi} \iint_{\Omega} \tilde{\chi}_{9}^{-} \sum_{k=1}^{3} \beta_{14 n}^{++} \partial_{2} \tilde{K}_{n, 4}^{2,+}\left(\boldsymbol{x}-\boldsymbol{x}^{0}, z_{2, n}^{+}\right) d x^{0}+\frac{1}{\pi} \sum_{k=5}^{6} \iiint_{\Omega} \tilde{\chi}_{13-k}^{-} \bar{F}_{k}^{+}\left(\boldsymbol{x}-\boldsymbol{x}^{0}\right) d \boldsymbol{x}^{0}=P_{4} \\
-\bar{R}_{14}^{+} \partial_{1} \tilde{\chi}_{9}^{-}+\frac{1}{\pi} \iint_{\Omega} \tilde{\chi}_{7}^{-}\left[\bar{S}_{24}^{+} \partial_{2}^{2} \frac{1}{r_{0}}-\bar{R}_{16}^{+} \partial_{1}^{2} \frac{1}{r_{0}}\right] d \boldsymbol{x}^{0}-\left[\bar{S}_{23}^{+}+\bar{R}_{15}^{+}\right] \frac{1}{\pi} \iint_{\Omega} \tilde{\chi}_{8}^{-} \partial_{12}^{2} d \boldsymbol{x}^{0}+ \\
+\frac{1}{\pi} \iint_{\Omega} \tilde{\chi}_{9}^{-} \sum_{k=1}^{3} \beta_{14 n}^{++} \partial_{2} \tilde{K}_{n, 3}^{2,+}\left(\boldsymbol{x}-\boldsymbol{x}^{0}, z_{2, n}^{+}\right) d \boldsymbol{x}^{0}+\frac{1}{\pi} \sum_{k=5}^{6} \iint_{\Omega} \tilde{\chi}_{13-k}^{-} \bar{F}_{k}^{+}\left(\boldsymbol{x}-\boldsymbol{x}^{0}\right) d \boldsymbol{x}^{0}=P_{5} .
\end{gathered}
$$

Given that

$\partial_{1}^{2} \frac{1}{r_{0}}=\frac{3}{2} \frac{\left(x_{1}-x_{1}^{0}\right)^{2}}{r_{0}^{5}}-\frac{1}{r_{0}^{3}}, \partial_{2}^{2} \frac{1}{r_{0}}=\frac{3}{2} \frac{\left(x_{2}-x_{2}^{0}\right)^{2}}{r_{0}^{5}}-\frac{1}{r_{0}^{3}}, \partial_{12}^{2} \frac{1}{r_{0}}=3 \frac{\left(x_{1}-x_{1}^{0}\right)\left(x_{2}-x_{2}^{0}\right)}{r_{0}^{5}}, \nabla^{2} \frac{1}{r_{0}}=\frac{1}{r_{0}^{3}}$.

And introducing the following operators:

$$
\begin{aligned}
& \Phi_{k}^{ \pm}[f](x, y)=\frac{1}{\pi} \iint_{\Omega} f(t, \tau) \frac{1}{r_{0}^{k}} d t d \tau, k=1,2,3, \\
& \Phi_{4}^{ \pm}[f](x, y)=\frac{1}{\pi} \iint_{\Omega} f(t, \tau) \frac{(x-t)(y-\tau)}{r_{0}^{5}} d t d \tau,
\end{aligned}
$$

$$
\Phi_{5}^{ \pm}[f](x, y)=\frac{1}{\pi} \iint_{\Omega} f(t, \tau) \frac{(x-t)^{2}}{r_{0}^{5}} d t d \tau ; \Phi_{6}^{ \pm}[f]\left(x_{1}, x_{2}\right)=\frac{1}{\pi} \iint_{\Omega} f\left(x^{0}\right) \frac{\left(x_{2}-x_{2}^{0}\right)^{2}}{r_{0}^{5}} d x^{0},
$$

we get:

$$
\begin{gathered}
\sum_{k=7}^{8} R_{2 k}^{+} \partial_{k-6} \tilde{\chi}_{k}^{-}\left(x_{1}, x_{2}\right)+R_{26}^{+} \Phi_{1}\left[\tilde{\chi}_{9}^{-}\right]\left(x_{1}, x_{2}\right)- \\
-\frac{1}{\pi} \sum_{k=5}^{7} \iint_{\Omega} \tilde{\chi}_{14-k}^{-} \sum_{n=1}^{3} \beta_{2 k n}^{++} \tilde{K}_{n, k+1}^{1,+}\left(\boldsymbol{x}-\boldsymbol{x}^{0}, \bar{z}_{2, n}^{+}\right) d \boldsymbol{x}_{0}=P_{3},
\end{gathered}
$$




$$
\begin{aligned}
-3\left[\bar{S}_{24}^{+}+\bar{R}_{16}^{+}\right] \Phi_{4}\left[\tilde{\chi}_{7}^{-}\right]\left(x_{1}, x_{2}\right)+\frac{3}{2}\left[\bar{S}_{23}^{+} \Phi_{5}\left[\tilde{\chi}_{8}^{-}\right]-\bar{R}_{15}^{+} \Phi_{6}\left[\tilde{\chi}_{8}^{-}\right]\right]-\bar{R}_{14}^{+} \partial_{2} \tilde{\chi}_{9}^{-}+ \\
+\frac{1}{\pi} \iint_{\Omega} \tilde{\chi}_{9}^{-} \sum_{k=1}^{3} \beta_{14 n}^{++} \partial_{2} \tilde{K}_{n, 4}^{2,+}\left(x-x^{0}, z_{2, n}^{+}\right) d x^{0}+\frac{1}{\pi} \sum_{k=5}^{6} \iint_{\Omega} \tilde{\chi}_{13-k}^{-} \bar{F}_{k}^{+}\left(\boldsymbol{x}-\boldsymbol{x}^{0}\right) d \boldsymbol{x}^{0}=P_{4}, \\
\quad-\bar{R}_{14}^{+} \partial_{1} \tilde{\chi}_{9}^{-}+\frac{1}{\pi} \iint_{\Omega} \tilde{\chi}_{7}^{-}\left[\bar{S}_{24}^{+} \partial_{2}^{2} \frac{1}{r_{0}}-\bar{R}_{16}^{+} \partial_{1}^{2} \frac{1}{r_{0}}\right] d \boldsymbol{x}^{0}-\left[\bar{S}_{23}^{+}+\bar{R}_{15}^{+}\right] \frac{1}{\pi} \iint_{\Omega} \tilde{\chi}_{8}^{-} \partial_{12}^{2} d \boldsymbol{x}^{0}+ \\
+\frac{1}{\pi} \iint_{\Omega} \tilde{\chi}_{9}^{-} \sum_{k=1}^{3} \beta_{14 n}^{++} \partial_{2} \tilde{K}_{n, 3}^{2,+}\left(\boldsymbol{x}-\boldsymbol{x}^{0}, z_{2, n}^{+}\right) d \boldsymbol{x}^{0}+\frac{1}{\pi} \sum_{k=5}^{6} \iint_{\Omega} \tilde{\chi}_{13-k}^{-} \bar{F}_{k}^{+}\left(\boldsymbol{x}-\boldsymbol{x}^{0}\right) d \boldsymbol{x}^{0}=P_{5} .
\end{aligned}
$$

Unknown functions are presented in the form:

$$
\begin{gathered}
\chi_{k}^{-}\left(x_{1}^{0}, x_{2}^{0}\right)=\varphi_{k}\left(x_{1}^{0}, x_{2}^{0}\right) \sqrt{Z\left(x_{1}^{0}, x_{2}^{0}\right),} \\
\varphi_{k}\left(x_{1}^{0}, x_{2}^{0}\right)=\sum_{i=0}^{N_{1}} \sum_{j=0}^{N_{2}} a_{i j}^{k}\left(x_{1}^{0}\right)^{i}\left(x_{2}^{0}\right)^{j},
\end{gathered}
$$

where $Z\left(x_{1}^{0}, x_{2}^{0}\right)$ is a geometric description of the $\partial \Omega$ crack boundary.

With an elliptical region rotated through the angle $\alpha$, the function $Z\left(x_{1}^{0}, x_{2}^{0}\right)$ can be defined:

$$
Z\left(x_{1}^{0}, x_{2}^{0}\right)=1-\left(\frac{\xi}{a}\right)^{2}-\left(\frac{\eta}{b}\right)^{2}=0,
$$

where $\xi=x_{1}^{0} \cos \alpha+x_{2}^{0} \sin \alpha$;

$\eta=x_{2}^{0} \cos \alpha-x_{1}^{0} \sin \alpha$.

To determine the unknown coefficients $a_{i j}^{k}, k=7,8,9$ system (24) is calculated at symmetrically located collocation points $\left(x_{n}, y_{n}\right) \in \Omega, n=\overline{1, M}$ :

$$
\begin{aligned}
& x_{n}=r_{s} \cos \left(\varphi_{t}\right), \\
& y_{n}=r_{s} \cos \left(\varphi_{t}\right),
\end{aligned}
$$

where $\varphi_{t}=\frac{\pi}{60} t, t=1,2, \ldots, 59$.

To take into account the strong influence of the polar angle on the stress intensity factor $r_{s}$, are chosen as the roots of the Chebyshev polynomial of the second kind, of the order of $n$ :

$$
n=26: U_{26}\left(r_{s}\right)=0, r_{s}=\cos \left(\frac{s+1}{27} \pi\right), s=\overline{0,12} .
$$

We obtain the expression for the stress intensity factor $[18,19,20,21]$ depending on the geometric parameters of the defect and the applied load.

Where the crack contour is described by a second-order curve $L\left(x_{1}^{0}, x_{2}^{0}\right)=A+B x_{1}^{0}+C\left(x_{1}^{0}\right)^{2}+D\left(x_{2}^{0}\right)^{2}$, where $A, B, C, D$ are the coefficients characterizing the shape of the curve (with an elliptical crack: $L(x)=\xi^{2} / a^{2}+\eta^{2} / b^{2}-1, \quad \xi=x_{1}^{0} \cos \alpha+x_{2}^{0} \sin \alpha$, $\left.\eta=x_{2}^{0} \cos \alpha-x_{1}^{0} \sin \alpha, C=1 / a^{2}, D=1 / b^{2}, A=-1, B=0\right)$, the expressions for the stress components in the plane of the crack have the form (terms containing a singularity are written out):

$$
\begin{aligned}
& \sigma_{33}(x)=\frac{R_{2,6}^{+}}{\pi} \iint_{\Omega} \chi_{9}^{-}\left(x_{0}\right) \frac{1}{r_{0}^{3}} d x_{0}, \\
& \sigma_{33}(x)=\frac{R_{2,6}^{+}}{\pi} \iint \chi_{9}^{-}\left(x_{0}\right) \frac{1}{r_{0}^{3}} d x_{0},
\end{aligned}
$$




$$
\sigma_{j 3}(x)=-\left[\bar{S}_{2,5-j}^{+}+\bar{R}_{1,7-j}^{+}\right] \frac{1}{\pi} \iint_{\Omega} \tilde{\chi}_{6+j}^{-} \partial_{12}^{2} \frac{1}{r_{0}} d x^{0}+\frac{1}{\pi} \iint_{\Omega} \tilde{\chi}_{9-j}^{-}\left[\bar{S}_{2, j+2}^{+} \partial_{1}^{2} \frac{1}{r_{0}}-\bar{R}_{1, j+4}^{+} \partial_{2}^{2} \frac{1}{r_{0}}\right] d x^{0}, \quad j=1,2 .
$$

By changing the variable $x_{1}^{0}=x_{1}+\rho \cos \theta, x_{2}^{0}=x_{2}+\rho \sin \theta, d x^{0}=\rho d \rho d \theta$ and performing the corresponding calculations, also considering that $k_{1}=\lim _{r \rightarrow 0} \sqrt{2 \pi r} \sigma_{3}$, where $r$ is the distance from the point $(\boldsymbol{x})$ to the point $\left(\boldsymbol{x}^{0}\right)$ of the crack contour, we obtain:

$$
k_{1}\left(x^{0}\right)=\frac{R_{2,6}^{+}}{\pi} \varphi_{9}\left(x_{1}^{0}, x_{2}^{0}\right) \Pi^{1 / 4}\left(x^{0}\right) ; \Pi\left(x^{0}\right)=\left(B+2 C x_{1}^{0}\right)^{2}+4 D^{2}\left(x_{2}^{0}\right)^{2} .
$$

From the found value of the stress intensity factor $k_{1}\left(x^{0}\right)$ and the value of the tensile stresses acting on the structure that forms the stresses $\sigma_{33}(x)$, one can determine the limiting value of the size of the major semi-axis of the elliptical defect $a$, at which this defect remains in equilibrium without the formation of the main crack from it.

Results and Discussion. The adequacy of the results obtained theoretically by determining the intensity of stresses in places of accumulation of inhomogeneities will be checked on the strength of welded joints of aluminum alloys [9], taking into account the occurrence of hot cracks in them. A comparative analysis of softening and the strength level of welded joints of plastic low-alloyed aluminum alloys and high-strength complex-alloyed aluminum alloys are carried out. The strain hardening of the joints of high-strength complex alloyed aluminum alloys obtained during argon-arc welding with a non-consumable electrode (AWNCE) provides a higher level of their strength than in fusion welding. In structures of critical use, exposed to significant force impact, high-strength complex alloyed aluminum alloys are used. For experimental studies, plastic low-alloyed aluminum alloys AMtsN, AMg2M, and AMg5M were used, and high-strength complex alloyed aluminum alloys AMg6M, 1420, 1201 and 1460. The mechanical properties of the welded sheets $1.8 \mathrm{~mm}$ thick are given in Table. 1.

Table 1

Mechanical properties of sheets of aluminum alloys with a thickness of $1.8 \mathrm{~mm}$ [7]

\begin{tabular}{c|c|c|c|c|c|c}
\hline \multicolumn{3}{|c|}{ Alloy grade } & \multirow{2}{*}{$\sigma_{\mathrm{v}}, \mathrm{MPa}$} & $\sigma_{0.2}, \mathrm{MPa}$ & $\delta, \%$ & $\alpha$, degrees \\
\hline ISO & EN & GOST & & & & \\
\hline AlMn1 & EN AW-3003 & AMtsN & 218 & 178 & 6 & 180 \\
\hline AIMg2 & AIMg2 & AMg2M & 176 & 88 & 23 & 180 \\
\hline- & - & AMg5M & 332 & 160 & 22 & 142 \\
\hline 5056 & - & AMg6M & 359 & 210 & 22 & 96 \\
\hline- & - & 1420 & 459 & 322 & 11 & 50 \\
\hline 2219 & - & 1201 & 423 & 303 & 12 & 60 \\
\hline- & - & 1460 & 565 & 523 & 9 & 36 \\
\hline
\end{tabular}

AWNCE was performed at a speed of $20 \mathrm{~m} / \mathrm{h}$ on an MW450 installation (Fronius, Austria) using the filler materials (Table 2). Standard samples were used from the welded joints obtained to determine the uniaxial tensile strength. The tests were carried out using the universal multi-purpose servohydraulic system MTS 810. The samples obtained by AWNCE were tested after removing the weld melt flush with the base metal, and with additionally cleaned their reinforcements, since these and other types of joints are used in welded structures. The softening of the metal in the welding zone was tested by measuring its hardness on a ROCKWELL device at a load of $600 \mathrm{~N}$ and a ball diameter of $1 / 16^{\prime \prime}$. For welded joints of plastic alloys AMtsN and AMg2M, the microhardness of the metal was measured using a PMT-3 microhardness tester with a load of $0.1 \mathrm{~N}$. The microstructure of the welded joints was studied using a MIM-8M optical microscope. Cross-sections of the compounds obtained by AWNCE were preliminarily prepared by electrolytic polishing and their additional etching in a solution of perchloric, nitric, and hydrofluoric acids. 
The conducted experimental studies made it possible to test softening, strength level, and structural features of the joints obtained by fusion welding. It was established that during AWNCE of plastic lowalloyed aluminum alloys AMtsN and $\mathrm{AMg} 2 \mathrm{M}$, the minimum hardness occurs in the metal, where the samples undergo tensile fracture, and in welded joints of the AMg5M alloy the minimum hardness is in the central part of the weld. Therefore, samples without reinforcement are destroyed along the seam, and with amplification, they are destroyed along the heataffected zone (HAZ).

It should be noted that the minimum values of metal hardness in the fracture zone of samples of welded joints of alloys AMtsN and AMg2M obtained by both welding methods are approximately the same, their tensile strength is at the level of 113 and $170 \mathrm{MPa}$, respectively (Table 3).

Table 3

Strength of welded joints of aluminum alloys obtained by various welding methods ${ }^{*}$

\begin{tabular}{c|c|c|c|c}
\hline \multirow{2}{*}{ Alloy grade } & \multicolumn{4}{|c}{ AWNCE } \\
\cline { 2 - 5 } & \multicolumn{2}{|c|}{ Reinforced Sample } & \multicolumn{2}{c}{ Non-reinforced Sample } \\
\cline { 2 - 5 } & $\sigma_{\mathrm{v}}, \mathrm{MPa}$ & Place of destruction & $\sigma_{\mathrm{v}}, \mathrm{MPa}$ & Place of destruction \\
\hline AMtsN & 113 & $\mathrm{HAZ}$ & 113 & HAZ \\
\hline AMg2M & 170 & - & 170 & - \\
\hline AMg5M & 320 & - & 300 & welding seam \\
\hline AMg6M & 345 & fusion zone & 324 & - \\
\hline 1420 & 373 & - & 320 & - \\
\hline 1201 & 296 & - & 239 & - \\
\hline 1460 & 311 & - & 257 & - \\
\hline
\end{tabular}

Only samples without reinforcement from AMg5M alloy obtained by AWNCE have lower (300 $\mathrm{MPa}$ ) strength and are destroyed in the central part of the weld, where the metal is characterized by minimal hardness. Therefore, structural changes in the weld metal and its fusion zones (FZ) with the base metal occurring during fusion welding of these alloys. practically do not affect the strength of welded seams.

So, when welding in the solid phase of the AMg6M alloy, the formation of fine grains in the weld leads to its strain hardening. Therefore, the destruction of such samples occurs according to the HAZ, where the metal hardness is minimal, and the strength is at the level of $336 \mathrm{MPa}$.

Non-reinforced Samples obtained by the AWNCE method break down along the seam and have a tensile strength of $324 \mathrm{MPa}$. The maximum strength (345 MPa) is characterized by reinforced specimens, which are destroyed by tension along the welded joint of the base metal.

In thermally hardened alloys (1420,1201, and 1460), the solid solution is in a supersaturated state. Therefore, because of thermomechanical action in the welds, besides grinding the grains, there is a softening of the metal, because of the release of excess intermetallic phases from the solid solution and their coagulation. Small hardening phases in the weld core are partially dissolved, and large lowmelting intermetallic inclusions are partially melted, forming a new solid solution.

\footnotetext{
* The average values of the indicators according to the test results of three to five samples are given
} 
In the welded seams of such alloys obtained by AWNCE, the minimum hardness of the metal is observed in the central part of the welds, where the samples are destroyed without reinforcement [7]. Here, the difference in strength substantially depends on the chemical composition of the alloy being welded. So, if for alloy 1420 it is only $22 \mathrm{MPa}$, then for alloys 1460 and 1201, respectively, 52 and 71 MPa. This is because of the peculiarities of the formation of seams with AWNCE.

The formation of a compound in the solid phase because of the movement of plasticized metal in a limited volume at an over-pressure contributes to grain refinement, an increase in the volume fraction of boundaries and crushing of intermetallic phases.

The samples with reinforcement obtained by AWNCE are always destroyed along the weld zone of the base metal and have higher strength than the samples without reinforcement, which are destroyed by the weld metal.

Strain hardening of seams occurring in high-strength complex alloyed aluminum alloys AMg6M, 1420, 1201 and 1460 obtained by AWNCE helps to reduce their strength.

Grinding of grains, an increase in the volume fraction of their boundaries and crushing of intermetallic phases in the joints at AWNCE. Aluminum alloys help to reduce their softening.

\section{Conclusions}

As a result of the research, methods for solving the scientific and technical problem are proposed, which consist in establishing design dependencies for determining the influence of hereditary defects formed in welded joints of structures on their crack resistance and creating technological requirements for the welding operation, taking into account accumulated damage and heterogeneities. The following tasks were solved:

1. The mechanism of the formation and development of defects such as cracks in welding joints of functionally gradient materials under the influence of thermomechanical phenomena accompanying the manufacturing technology and operation of structural elements is studied.

2. Mathematical models have been developed that describe the mechanical processes in the weld of structural elements, taking into account heterogeneities that affect the formation of cracks, which helps to reduce the bearing capacity of such elements.

3. The adequacy of the constructed model was checked on high-strength complex alloyed aluminum alloys obtained by argon-arc welding with a non-consumable electrode.

\section{Література}

1. Промышленные алюминиевые сплавы: Справочник / С.Г. Алиева и др. ; под редак. Белов А.Ф. и др. М. : Металлургия, 1984. 528 с.

2. .Рабкин Д.М., Игнатьев В.Г., Довбищенко И.В. Состояние и перспективы применения алюминиевых сплавов в сварных конструкциях. Киев : Наук. думка, 1975. 48 с.

3. Колобнев Н.И., Фридляндер И.Н. Алюминий-литиевые сплавы - новый этап снижения массы летательных аппаратов. Авиационные материалы на рубеже XX-ХХІ веков. 1994. С. 89-92.

4. Алюминий и его сплавы в современных сварных конструкциях / Ищенко А.Я., Лабур Т.М., Бернадский В.Н., Маковецкая О.К. Киев : Екотехнологія, 2006. 112 с.

5. Овчинников В.В., Дриц А.М., Крымова Т.В. Технологические особенности производства сварных конструкций летательных аппаратов из алюминиево-литиевого сплава 1460. Сварочное производство. 1997. № 12. С. 26-29.

6. Dawes C.J. An introduction to friction stir welding and its development. Welding \& Metal Fabrication. 1995. № 1. Р. 13-16.

7. Покляцкий А.Г., Ищенко А.Я., Яворская М.Р. Прочность сварных соединений алюминиевых сплавов при сварке трением с перемешиванием. Проблеми ресурсу $і$ безпеки експлуатації конструкиій, споруд та машин / За ред. Б. С. Патона. Київ : IE3 ім. Є.О. Патона, 2006. С. 365-367.

8. Kallee S., Nicholas D. Causing a stir in the future. Welding and Joining. 1998. № 2. P. 18-21.

9. Ищенко А.Я., Подъельников С.В., Покляцкий А.Г. Сварка трением с перемешиванием алюминиевых сплавов (Обзор). Автоматическая сварка. 2007. № 11. С. 32-38.

10. Kunitsyn M.V., Usov A.V. Tribocorrosion research of NI-Al2O3/TIO2 composite materials obtained by the method of electrochemical deposition. Сучасні техноглогіï в машинобудуванні. 2017. № 12. P. 61-70. 
11. Морозов Н.Ф. Математические вопросы теории трещин. Москва : Наука. 1984. 256 с.

12. Попов Г.Я. Избранные труды. Том 1, 2. Одесса : Издат.-полиграф. дом ВМВ, 2007. 896 с.

13. Екобори Т. Научные основы прочности и разрушения материалов. Пер. с яп. Киев .: Наукова думка, 1978. 352 с.

14. Попов Г.Я. Концентрация упругих напряжений возле штампов, разрезов, тонких включений и подкреплений. Москва : Наука, 1982. 344 с.

15. Усов А.В. Математическое моделирование процессов контроля покрытия элементов конструкций на базе СИУ. Проблемы машиностроения. 2010. Т. 13, №1. С. 98-109.

16. Моделирование систем: монография / Оборский Г.А., Дащенко А.Ф., Усов А.В., Дмитришин Д.В. Одесса : Астропринт, 2013. 664 с.

17. Введение в методы оптимизации и теорию технических систем / Усов А.В., Оборский Г.А., Морозов Ю.А., Дубров К.А. Одесса : Астропринт, 2005. 496 с.

18. Райс Дж. Математические методы в механике разрушения. Разрушение. Москва : Мир, 1975. T. 2. C. 204-335.

19. Черепанов Г.П. Механика хрупкого разрушения. Москва : Наука, 1974. 640 с.

20. Витвицкий П.М., Попина С.Ю. Прочность и критерии хрупкого разрушения стохастически дефектных тел. Киев : Наук. Думка, 1980. 187 с.

21. Саврук М.П., Осив П.Н., Прокопчук И.В. Численный анализ в плоских задачах теории трещин. Киев : Наукова думка, 1989. 248 с.

\section{References}

1. Aliyeva, S.G. et al. (1984). Industrial Aluminum Alloys: Handbook. In Belov A. F. et al. (Eds.). Moscow: Metallurgiya.

2. Rabkin, D.M., Ignatiev, V.G., \& Dovbishchenko, I.V. (1975). State and prospects of the use of aluminum alloys in welded structures. Kiev: Naukova Dumka.

3. Kolobnev, N.I., \& Fridlyander, I.N. (1994). Aluminum-lithium alloys - a new stage in reducing the weight of aircraft. Aviation materials at the turn of the 20th - 21st centuries: Collection of scientific papers, 89-92.

4. Ishchenko, A.Ya., Labur, T.M., Bernadsky, V.N., \& Makovetskaya, O.K. (2006). Aluminum and its alloys in modern welded structures. Kiev: Ekotehnologiya.

5. Ovchinnikov, V.V., Drits, A.M., \& Krymova, T.V. (1997). Technological features of the production of welded structures of aircraft from aluminum-lithium alloy 1460. Welding production, 12, $26-29$.

6. Dawes, C.J. (1995). An introduction to friction stir welding and its development. Welding \& Metal Fabrication, 1, 13-16.

7. Poklyatsky, A.G., Ischenko, A.Ya., \& Yavorskaya, M.R. (2006). Strength of welded joints of aluminum alloys during friction welding with stirring. In Paton B.E. (Eds.). Problems to the resource and safety design, equipment of machines (pp. 365-367). IEZ im. Patona E.O.

8. Kallee, S., \& Nicholas, D. (1998). Causing a stir in the future. Welding and Joining, 2, 18-21.

9. Ischenko, A.Ya., Podel'nikov, S.V., \& Poklyatsky, A.G. (2007). Friction welding with stirring of aluminum alloys (Review). Automatic welding, 11, 32-38.

10. Kunitsyn, M.V., \& Usov, A.V. (2017). Tribocorrosion research of NI-Al2O3/TIO2 composite materials obtained by the method of electrochemical deposition. Modern technologies in mechanical engineering, 12, 61-70.

11. Morozov, N.F. (1984). Mathematical problems in the theory of cracks. Moscow: Nauka.

12. Popov, G.Ya. (2007). Selected Works. Volume 1, 2. Odessa: Izdatel'sko-poligraficheskiy dom VMV.

13. Ekobori, T. (1978). Scientific foundations of strength and fracture of materials. Tran. from Jap. Kiev: Naukova Dumka.

14. Popov, G.Ya. (1982). Elastic stress concentration near dies, cuts, thin inclusions and reinforcements. Moscow: Nauka.

15. Usov, A.V. (2010). Mathematical modeling of control processes for the coating of structural elements based on SIE. Engineering problems, 13(1), 98-109.

16. Oborsky, G.A., Dashchenko, A.F., Usov, A.V., \& Dmitrishin, D.V. (2013). Modeling systems: monograph. Odessa: Astroprint.

17. Usov, A.V., Oborsky, G.A., Morozov, Yu.A., \& Dubrov, K.A. (2005). Introduction to optimization methods and theory of technical systems. Odessa: Astroprint.

18. Rice, J. (1975). Destruction. Mathematical methods in fracture mechanics (vol. 2, pp. 204-335). Mir.

19. Cherepanov, G.P. (1974). Mechanics of brittle fracture. Moscow: Nauka. 
20. Vitvitsky, P.M., \& Popina, S.Yu. (1980). Strength and criteria for brittle fracture of stochastically defective bodies. Kiev: Naukova Dumka.

21. Savruk, M.P., Osiv, P.N., \& Prokopchuk, I.V. (1989). Numerical analysis in plane problems of crack theory. Kiev: Naukova Dumka.

Усов Анатолій Васильович; Usov Anatoly, ORCID: https://orcid.org/0000-0002-3965-7611

Морозов Юрій Олександрович; Morozov Yurii, ORCID: https://orcid.org/0000-0003-4027-2353

Куніцин Максим Володимирович; Kunitsyn Maksym, ORCID: https://orcid.org/0000-0003-1764-8922

Тонконоженко Анатолій Мстиславович; Tonkonozhenko Anatoliy, ORCID: https://orcid.org/0000-0002-5953-4950

Черниш Ігор Олександрович; Chernush Ihor, ORCID: https://orcid.org/0000-0002-8473-3505

Received January 12, 2020

Accepted February 19, 2020 